\title{
Influence of Phosphorous, Nitrogenous Fertilizers and Seeding Rates on Green Forge Yield and Its Quality of Sudangrass (Sorghum vulgare var. Sudanense (Piper) Stapf) under Ismailia Governorate Conditions - Egypt
}

\author{
Awad, A. ${ }^{1}$; S. I. Hafiz ${ }^{1}$; M. S. Hammada ${ }^{1}$; S. El-Hendawy ${ }^{1 \& 2}$; E. A. Ali ${ }^{1}$ and Azza M. A. M. El-Nouby ${ }^{1}$
}

1. Agronomy Department, Faculty of Agriculture, Suez Canal University, 41522 Ismailia, Egypt

2. Plant Production Department, College of Food and Agriculture Sciences, King Saud University, Saudi Arabia

\section{Received: $3 / 6 / 2014$}

\begin{abstract}
Two field experiments were conducted in summer seasons of 2010 and 2011 at Agricultural Research Station, Faculty of Agriculture; Suez Canal University at Ismailia governorate; Egypt. The soil textures of experiments were sandy. The experiments are aimed to study the response of Sudangrass cv. Giza "2" to three phosphorus fertilizer rates $(0,37$ and $74 \mathrm{~kg} \mathrm{P2O5} / \mathrm{ha})$, three rates of nitrogen $(74,121$ and $171 \mathrm{~kg} \mathrm{~N} / \mathrm{ha})$, and two seeding rates (24 and $36 \mathrm{~kg}$ $\mathrm{seed} / \mathrm{ha}$ ). The design of each experiment was split-split plots design. Sowing date was 9th of May in two growing seasons. Plant height, leaves and stem fresh weight/plant, total plant fresh weight, leaf-stem ratio, green forage yield/cut, total green forage yield/season, $\mathrm{CP} \%$, and $\mathrm{CP}$ yield/cut were increased by increasing phosphorus fertilizer rates up to $74 \mathrm{kgP} 2 \mathrm{O} 5 / \mathrm{ha}$, and also, it were increased by increasing nitrogen fertilizer rates up to $171 \mathrm{~kg} \mathrm{~N} / \mathrm{ha}$, while crude fiber \% was increased by increasing phosphorus levels and decreased by increasing nitrogen levels. At all cuts, increase seeding rates up to $36 \mathrm{~kg} / \mathrm{ha}$ significantly increased plant height, green forage yield/cut and total green forage yield/season, but decreased significantly leaves and stem fresh weight/plant, total plant fresh weight, but didn't affect significantly leaf-stem ratio, $\mathrm{CP}$ yield/cut, and $\mathrm{CF} \%$.
\end{abstract}

Keywords: Sudangrass- sorghum- phosphorus fertilization - nitrogenous fertilization- seeding rates.

\section{INTRODUCTION}

Green forage yield of sorghum (Sorghum bicolor var. Sudanense) is an important forage crop in tropical, semitropical and even warm-temperate regions (Bahrani and Ghenateghestani (2004). The shortage of green forage in summer season is considered to be one of main problem in feeding animals in Egypt. Therefore, it was necessary to set a plan for improve forage yield and quality characteristics of Sudangrass, which is considered an important summer forage crop. Agricultural operations are considered one of the main objectives to achieve an increase in forage yield and crop quality. Nitrogen fertilizer is considered one of main nutrients to be most important factor to increase productivity of forage crops. This element can play an important role in some physiological operation such as photosynthesis rates of crop leaves, amino and nucleic acids assimilation. Therefore, if nitrogen is a limiting factor during active reproductive phase a reduction in forage yield and its quality will be occurred. Increasing nitrogen fertilizer significantly increase biomass above ground of sorghum cultivars (Almodares, et al., 2009; El-Sarag and Abu Hashem 2009; Yagoub and Abdelsalam (2010); Dorde, et al., 2011 and Afzal, et al., 2012 and 2013).

Green forage yield was increased linearly with increasing nitrogen fertilization rates (Zahid et al, 2002). Afzal, et al., (2013) found that total green forage yield of three cuttings were differed significantly among the different nitrogen levels $(0,28.75,45$ and $57.5 \mathrm{~kg}$ $\mathrm{N} /$ ha. Nitrogen fertilizer increased crude protein present increased while crude fiber present decreased. (Almodares, et al., 2009; El-Sarag and Abu Hashem, 2009; Dorde, et al., 2011); and Afzal, et al., (2013) in Pakistan, showed that application of $100 \mathrm{~kg} \mathrm{~N} / \mathrm{acre}$, and the highest value of crude protein \% $(12.0 \%)$ was observed by $100 \mathrm{~kg}$ N/acre followed by $75 \mathrm{~kg} \mathrm{~N} /$ acre
$(10.6 \%)$ while, the minimum crude protein content $(7.5 \%)$ was observed by zero nitrogen per acre.

The role of phosphorus is not conspicuous but it might have played a prominent role to increase rate of biochemical processes which are occurring in the plant to enhance plant growth (Zahid etal, 2002). Sorghum is response to addition of phosphorus especially soils has very low available phosphorus (Yoana, et al., 2010). Phosphorus is critical for early development of young sorghum plant. A phosphorus deficiency can cause a restricted of root development and delayed flowering and maturity in sorghum (Mahmood, 2012). Mahmud, (2003) resulted that gradual increase in plant height and fodder yield also of two sorghum cultivars (Hegari and JS 263) was observed with the increase in phosphorus rates up to $100 \mathrm{~kg} \mathrm{P}_{2} \mathrm{O}_{5} /$ ha. A progressive increase in crude protein content, crude fiber was observed with the increase in phosphorus rates up to $100 \mathrm{~kg} \mathrm{P}_{2} \mathrm{O}_{5} / \mathrm{ha}$. Roy and Khandaker (2010) concluded that the tallest plant was found with application of $80 \mathrm{~kg}$ tri super phosphate (TSP)/ha and the shortest plant was at control group. Highest green fodder yield was observed at $40 \mathrm{~kg}$ $\mathrm{TSP} /$ ha followed by $80 \mathrm{~kg} \mathrm{TSP} / \mathrm{ha}$ followed by zero $\mathrm{kg}$ $\mathrm{TSP} / \mathrm{ha}$ in first cutting. It may be suggested that sorghum fodder can be cultivated through the application of $80 \mathrm{~kg}$ (TSP) phosphorus fertilizer and harvested at the age of 66 days at first cutting for maximum production.

John, et al., (2012) concluded that plant height of sorghum has increased with increasing seeding rates whereas, low seeding rates (116.000 seeds/ha) was preferable because higher seeding rates don't positively effect yield and may cause morphological changes (i.e. taller plants with thinner stems) conductive to lodging) In Pakistan Mahmood (2012) observed that higher plant density of sorghum bicolor $\left(16,24\right.$ and 32 plants $\left.\mathrm{m}^{-2}\right)$ caused an increase in plant height, while plant density didn't cause a clear change in protein concentration 
(7.8, 7.5 and $7.9 \%$ for the three seeding rates, respectively). Bahrani and Ghenateghestani (2004) reported that increasing plant density $(25,33$ and 50 plant $/ \mathrm{m}^{2}$ ) was decreased crude protein percentage of Sorghum bicolor L. Moench var. Sudanense. Therefore, the objective of this study was to evaluate the impact of different agronomic practices (phosphorus fertilizer levels, nitrogen fertilizer rates and different seeding rates) on quantity and quality of forage yield of Sudangrass. Our results are expected to be useful for assessing the optimal range for these factors in terms of forage production in Sudan grass, especially in arid and semiarid regions.

\section{MATERIALS AND METHODS}

Two field experiments were conducted to study the response of Sudangrass cv. Giza "2' as forage crop to three levels of each phosphorus and nitrogen fertilizer and two seeding rates. The experiments were carried out in Agricultural Research Station, Faculty of Agriculture; Suez Canal University at Ismailia governorate; Egypt, during summer seasons of 2010 and 2011. The soil texture of the experimental site is predominantly sandy as shown in Table (1) which explains mechanical and chemical analysis of experimental site in two successive growing seasons. Geographical location of the Agricultural Research Station is located at $30^{\circ} 58^{\prime} \mathrm{N}$, $32^{\circ} 23^{\prime} \mathrm{E}$ and located at a height of 13 meters above sea level (m.a.s.l). The climate of Suez Canal Region is hot and dry from May to October months, and temperatures could be reached up to $40{ }^{\circ} \mathrm{C}$. The growing season of Sudangrass is during this period. Meteorological data for two growing seasons are shown in Table (2). Sudangrass Giza-2 variety was selected and tested at two seeding rates 24 and $36 \mathrm{~kg} / \mathrm{ha}$. Nitrogenous fertilizer was tested at three rates; 74.121 and $171 \mathrm{~kg}$ $\mathrm{N} /$ ha as ammonium sulfate $(20.5 \% \mathrm{~N})$ while Phosphorus fertilizer as calcium monophosphate $\left(15.5 \% \mathrm{P}_{2} \mathrm{O}_{5}\right)$ was used at three rates 0,37 and $74 \mathrm{~kg} \mathrm{P}_{2} \mathrm{O}_{5} / \mathrm{ha}$. A randomized complete block split-split plot design with three replications was used in each season. Each experimental plot contained 6 rows $50 \mathrm{~cm}$ apart and three meters in length $\left(9 \mathrm{~m}^{2}\right.$ in total area). Sudangrass cv. Giza "2' seeds were drilled handily in rows on $9^{\text {th }}$ of May in both growing seasons 2010 and 2011. In previous winter season the experimental soil sites were fallow. All other agronomic practices were applied as recommended for this crop. Three cuts were taken during each growing season; first cut was taken at 60 days after sowing, the second cut at 50 days from the first one and third cut was taken after 45 days from the second one. At each cut five surrounded plants were taken randomly from outer two rows to determine vegetative growth parameter as an average per plant. The growth parameters were as follows: Plant height (cm), Leave fresh weight ( $\mathrm{g} / \mathrm{plant})$, Stems fresh weight (g/plant), Total plant fresh weight (g), leaves/stem ratio; Fresh forage yield per cut (ton/ha); Total fresh forage yield (ton/ha/season); crude protein percentage and crude fiber percentage per cut was determined according to method to Sadasivan and Manickam (1991)
All measurements in this study were analyzed by using an analysis of variance (ANOVA) appropriate for a randomized complete block split-split plot design. Comparisons among averages of each factor were accomplished by using LSD test or Duncan's (1955) multiple tests. Statistical analysis was done by using the COSTAT system for Windows, version 6.311 (Cohort Software, Berkeley, CA, USA).

\section{RESULTS AND DISCUSSIONS}

\section{Effect of phosphorus fertilizer levels;}

Data in table (3) indicate that plant height, leaves and stem fresh weights per plant, total plant fresh weight and leaf-stem ratio were affected significantly by increasing phosphorus fertilization rates up to $74 \mathrm{~kg}$ $\mathrm{P}_{2} \mathrm{O}_{5} /$ ha. Green forage yield per cut also, was increased significantly by increasing phosphorus fertilization rates up to $74 \mathrm{~kg} \mathrm{P}_{2} \mathrm{O}_{5} / \mathrm{ha}$ at all cuts of two growing seasons (table 6), and difference between rates of 37 and $74 \mathrm{~kg}$ $\mathrm{P}_{2} \mathrm{O}_{5} /$ ha was insignificant at first cut in two growing seasons. Total green forage yield/ season was increased significantly by increasing phosphorus fertilization rates up to $74 \mathrm{~kg} \mathrm{P}_{2} \mathrm{O}_{5} / \mathrm{ha}$ in the two seasons, this trait was increased from $72.77,78.13$ to $96.94 \mathrm{t} /$ ha at first season and $74.44,80.19$ to $88.71 \mathrm{t} / \mathrm{h}$ a at second season for three phosphorus rates, respectively (Table 6).

Crude protein percentage (Table 6) was increased significantly by increasing phosphorus fertilization rates up to $74 \mathrm{~kg} / \mathrm{ha}$, but difference between rate of 37 and 74 $\mathrm{kg} \mathrm{P}_{2} \mathrm{O}_{5} /$ ha was insignificant at all cuts of two growing seasons. While, difference between rates of zero and 37 $\mathrm{kg} \mathrm{P}_{2} \mathrm{O}_{5} /$ ha was insignificant at all cuts, except at first and third cuts in the second season. Crude protein yield/cut was increased significantly by increasing phosphorus fertilization rates up to $74 \mathrm{~kg} / \mathrm{ha}$. Also, Crude fiber percentage was increased significantly by increasing phosphorus fertilization rates up to $74 \mathrm{~kg} / \mathrm{ha}$, but difference between rate 37 and $74 \mathrm{~kg} \mathrm{P}_{2} \mathrm{O}_{5} / \mathrm{ha}$ was significant of first cut at first growing season. While, the differences between the rate 0 (zero) and $37 \mathrm{~kg} \mathrm{P}_{2} \mathrm{O}_{5} /$ ha was significant at all cuts of second season (Table 6). The obtained results of this investigation are in accordance with those obtained by Hassan (1976); Haggag et al (1986); Hafiz and El-Kholy, (2000); zahid, et al (2002); Mahmud (2003), and Mahmood, 2012).

\section{Effect of nitrogenous fertilizer;}

Data in table (4) show that plant height, leaves fresh weight per plant, stems fresh weight per plant, fresh leaf-stem ratio and total plant fresh weight were increased significantly with increasing nitrogen fertilizer rates up to $171 \mathrm{~kg} \mathrm{~N} / \mathrm{ha}$ at all cuts in the two growing seasons. Green forage yield per cut was increased significantly by increasing nitrogen fertilizer rates up to $171 \mathrm{~kg} \mathrm{~N} / \mathrm{ha}$. Total green forage yield/ season was increased significantly by increasing nitrogen fertilizer rates up to $171 \mathrm{~kg} \mathrm{~N} / \mathrm{ha}$ in the two seasons, where total green forage yield was recorded at first season 70.61, 83.45 and $93.76 \mathrm{t} / \mathrm{ha}$ and $68.29,80.13$ and $94.91 \mathrm{t} / \mathrm{ha}$ at second season for three nitrogen fertilizer rates in the two seasons, respectively (Table 7). Crude protein percentage and crude protein yield per 
cutting at all cuts in the two growing seasons were increased by increasing nitrogen fertilizer rates up to $171 \mathrm{~kg} \mathrm{~N} /$ ha increased significantly (table 7). Increasing nitrogen fertilizer rates up to $171 \mathrm{~kg}$ nitrogen/ha decreased crude fiber percentage at second cut of first season and first beside to third cut of second season. The results of this study are compatible with results of other investigators such as Zahid etal, (2002); Almodares, et al., 2009; El-Sarag and Abu Hashem 2009; Yagoub and Abdelsalam (2010) Dorde, et al., 2011; Afzal, et al., 2012 and Afzal, et al., (2013)

\section{Effect of seeding rates}

Data in table (5) cleared that plant height was increased by increasing seeding rate from 24 to $36 \mathrm{~kg}$ seeds/ha at three cuts in two growing season. While, leaves fresh weight per plant, stems fresh weight per plant and total plant fresh weight were increasing by decreasing seeding rate from 36 to $24 \mathrm{~kg}$ seeds/ha at the three cuttings in the two growing season, while leafstem ratio wasn't affected significantly by seeding rates at all cuts in the two growing successive seasons (Table 5).

Green forage yield/cut was increased significantly by increasing seeding rate from 24 to $36 \mathrm{~kg}$ seeds/ha at three cuts in the two growing season. The higher seeding rate recorded the highest weight of fresh forage yield per cut than the lower one. Green forage yield per cut from using $36 \mathrm{~kg}$ seeds/ha overcome that from using $24 \mathrm{~kg}$ seeds/ha by $4.64,3.99$ and $5.13 \mathrm{t} / \mathrm{ha}$ for three cuts of first season, respectively and by $3.84,4.46$ and 4.68 $\mathrm{t} /$ ha for three cuts, respectively in second season (Table 8).

Total green forage yield was increased significantly by increasing seeding rate from 24 to $36 \mathrm{~kg}$ seeds/ha in two seasons. The higher seeding rate increased total green forage yield by 12.35 and $18.44 \%$ at first and second season, respectively (table 8).

Table (1): The mechanical and chemical analysis of soil at the experimental site in two growing seasons (2010 and 2011).

\begin{tabular}{|c|c|c|c|}
\hline Properties & Over two seasons & Properties & Over two seasons \\
\hline \multicolumn{2}{|c|}{ Particle size distribution (\%) } & \multicolumn{2}{|l|}{ Soluble anions $\left(\mathrm{meqL}^{-1}\right)$} \\
\hline Sand & 93.12 & $\left(\mathrm{CO}_{3}\right)^{-2}$ & 0.00 \\
\hline Silt & 2.38 & $\left(\mathrm{HCO}_{3}\right)^{-}$ & 4.75 \\
\hline Clay & 4.50 & $\mathrm{Cl}^{-}$ & 9.50 \\
\hline Texture class & Sandy & $\left(\mathrm{SO}_{4}\right)^{-2}$ & 9.66 \\
\hline Soil order & 1.55 & Organic $\mathrm{C}\left(\mathrm{g} \mathrm{kg}^{-1}\right)$ & 1.72 \\
\hline Field capacity $(\%)$ & 17.20 & Total N $\left(\mathrm{g} \mathrm{kg}^{-1}\right)$ & 0.16 \\
\hline $\mathrm{pH}$ & 8.19 & Available N ( $\left.\mathrm{mg} \mathrm{kg}^{-1}\right)$ & 5.50 \\
\hline $\mathrm{ECe}\left(\mathrm{dSm}^{-1}\right)$ & 2.35 & Available $\mathrm{P}\left(\mathrm{mg} \mathrm{kg}^{-1}\right)$ & 1.17 \\
\hline \multicolumn{2}{|c|}{ Soluble cations $\left(\mathrm{meq}^{-1}\right)$} & \multicolumn{2}{|l|}{ Micronutrients $\left(\mathrm{mg} \mathrm{kg}^{-1}\right)$} \\
\hline $\mathrm{Ca}^{+2}$ & 11.50 & $\mathrm{Fe}$ & .68 \\
\hline $\mathrm{Mg}^{+2}$ & 7.50 & $\mathrm{Mn}$ & 1.16 \\
\hline $\mathrm{Na}^{+}$ & 3.93 & $\mathrm{Zn}$ & 1.23 \\
\hline $\mathrm{K}^{+}$ & 0.98 & $\mathrm{Cu}$ & 0.24 \\
\hline
\end{tabular}

Source: Water and Soil Department, Agriculture Faculty, Suez Canal University.

Table (2): The Meteorological data of air and soil at the experimental site in two growing seasons (2010 and 2011$)$.

\begin{tabular}{|c|c|c|c|c|c|c|c|c|}
\hline \multirow{3}{*}{$\begin{array}{l}\text { Meteorological data } \\
\text { Season }\end{array}$} & \multicolumn{4}{|c|}{ Air tempera $\left({ }^{\circ} \mathrm{C}\right)$} & \multicolumn{4}{|c|}{ Air moisture \% } \\
\hline & \multicolumn{2}{|c|}{2010} & \multicolumn{2}{|c|}{2011} & \multicolumn{2}{|c|}{2010} & \multicolumn{2}{|c|}{2011} \\
\hline & $\max$ & $\min$ & $\max$ & $\min$ & $\max$ & $\min$ & $\max$ & $\min$ \\
\hline$* 1^{\text {st }}$ cutting & 33.4 & 19.2 & 33.8 & 20.0 & 82.1 & 24.6 & 89.6 & $\overline{28.2}$ \\
\hline$* 2^{\text {nd }}$ cutting & 34.9 & 23.5 & 37.5 & 23.9 & 85.3 & 35.2 & 89.0 & 28.3 \\
\hline$* 3^{\text {rd }}$ cutting & 32.8 & 20.4 & 35.7 & 21.3 & 85.2 & 31.2 & 88.6 & 28.1 \\
\hline \multirow[t]{2}{*}{ Meteorological data } & \multicolumn{4}{|c|}{ soil tempera at $10 \mathrm{~cm}$ depth } & \multicolumn{4}{|c|}{ soil tempera at $15 \mathrm{~cm}$ depth } \\
\hline & $\max$ & $\min$ & $\max$ & $\min$ & $\max$ & $\min$ & $\max$ & $\min$ \\
\hline$* 1^{\text {st }}$ cutting & 35.3 & 24.4 & 33.0 & 24.3 & 31.3 & 27.5 & 27.7 & 24.6 \\
\hline$* 2^{\text {nd }}$ cutting & 38.3 & 29.2 & 35.9 & 27.0 & 27.7 & 24.6 & 28.5 & 26.0 \\
\hline$* 3^{\text {rd }}$ cutting & 35.0 & 26.6 & 34.5 & 24.8 & 27.7 & 24.6 & 27.7 & 24.6 \\
\hline
\end{tabular}

\begin{tabular}{lcccccc} 
& \multicolumn{3}{c}{ Soil tempera at 5 cm depth } & \multicolumn{2}{c}{ Evaporation\% } \\
\cline { 2 - 7 } & $\mathbf{m a x}$ & min & max & min & $\mathbf{2 0 1 0}$ & $\mathbf{2 0 1 1}$ \\
\hline$* \mathbf{1}^{\text {st }}$ cutting & 48.27 & 20.0 & 45.8 & 20.0 & 4.0 & 5.7 \\
$* 2^{\text {nd }}$ cutting & 55.4 & 23.9 & 51.4 & 21.7 & 4.4 & 7.9 \\
$* 3^{\text {rd }}$ cutting & 46.9 & 21.4 & 40.0 & 17.3 & 4.0 & 7.1 \\
\hline
\end{tabular}

$* 1^{\text {st }}$ cutting $=60$ days from $9 / 5$ to $7 / 7 \& * 2^{\text {nd }}$ cutting $=50$ days from $8 / 7$ to $26 / 8 \& * 3^{\text {rd }}$ cutting $=45$ days from $27 / 8$ to $10 / 10$

Source: Ministry of Agriculture and Land Reclamation, ARC, ARE. 
Crude protein percentage was increased by decreasing seeding rate from 36 to $24 \mathrm{~kg}$ seeds/ha, but this increases were significant only in first cut of first season and second cut in second season. While, crude protein yield per cut and crude fiber percentage were increased by increasing seeding rate from 24 to $36 \mathrm{~kg}$ seeds/ha., but these increases were significant only in second and third cuttings in second season only. (Table 8). These results are in accordance with those obtained by (Ahmed (2004) and Bahrani and Ghenteghestani (2004).

Effect of the interaction among phosphorus: nitrogenous and seeding rates:

The effect of the third interaction among the three studied factors on all studied characters wasn't significant at all cuts in the two growing seasons.

Table (3): Effect of phosphorus rates (kg/ha.) on vegetative characteristics of Sudangrass in two growing seasons (2010 and 2011).

\begin{tabular}{|c|c|c|c|c|c|c|}
\hline \multirow{2}{*}{$\begin{array}{l}\text { Season } \\
\text { Cutting No. }\end{array}$} & \multicolumn{3}{|c|}{ Season 2010} & \multicolumn{3}{|c|}{ Season 2011} \\
\hline & $1^{\text {st }}$ cut & $2^{\text {nd }}$ cut & $3^{\text {rd }}$ cut & $1^{\text {st }}$ cut & $2^{\text {nd }}$ cut & $3^{\text {rd }}$ cut \\
\hline \multicolumn{7}{|c|}{ 1- Plant height } \\
\hline $0 \mathrm{~kg} \mathrm{P}_{2} \mathrm{O}_{5} / \mathrm{ha}$ & $192.33 \mathrm{a}$ & $173.00 \mathrm{~b}$ & $145.56 \mathrm{~b}$ & $221.39 \mathrm{a}$ & $215.28 \mathrm{~b}$ & $141.11 \mathrm{~b}$ \\
\hline $37 \mathrm{~kg} \mathrm{P}_{2} \mathrm{O}_{5} / \mathrm{ha}$ & $206.22 \mathrm{a}$ & $186.11 \mathrm{ab}$ & $174.17 \mathrm{a}$ & $238.33 \mathrm{a}$ & $226.67 \mathrm{a}$ & $143.33 \mathrm{~b}$ \\
\hline $74 \mathrm{~kg} \mathrm{P}_{2} \mathrm{O}_{5} / \mathrm{ha}$ & $208.33 \mathrm{a}$ & $201.39 \mathrm{a}$ & $179.06 \mathrm{a}$ & $243.89 \mathrm{a}$ & $230.83 \mathrm{a}$ & $159.17 \mathrm{a}$ \\
\hline \multicolumn{7}{|c|}{ 2- Leaves fresh wt/plant (g) } \\
\hline 0 kg $\mathrm{P}_{2} \mathrm{O}_{5} / \mathrm{ha}$ & $41.78 \mathrm{c}$ & $36.72 b$ & $27.00 \mathrm{~b}$ & $48.33 \mathrm{~b}$ & $40.44 \mathrm{c}$ & $36.67 \mathrm{a}$ \\
\hline $37 \mathrm{~kg} \mathrm{P}_{2} \mathrm{O}_{5} / \mathrm{ha}$ & $48.33 \mathrm{~b}$ & $41.67 b$ & $32.83 \mathrm{~b}$ & $55.83 \mathrm{ab}$ & $47.44 \mathrm{~b}$ & $38.33 \mathrm{a}$ \\
\hline $74 \mathrm{~kg} \mathrm{P}_{2} \mathrm{O}_{5} / \mathrm{ha}$ & $54.17 \mathrm{a}$ & $48.33 \mathrm{a}$ & $38.61 \mathrm{a}$ & $58.61 \mathrm{a}$ & $52.28 \mathrm{a}$ & $40.56 \mathrm{a}$ \\
\hline \multicolumn{7}{|c|}{ 3- Stems fresh wt/plant (g) } \\
\hline 0 kg $\mathrm{P}_{2} \mathrm{O}_{5} / \mathrm{ha}$ & $133.61 \mathrm{a}$ & $131.83 \mathrm{a}$ & $97.22 \mathrm{c}$ & $150.11 \mathrm{~b}$ & $128.89 \mathrm{~b}$ & $130.78 \mathrm{a}$ \\
\hline $37 \mathrm{~kg} \mathrm{P}_{2} \mathrm{O}_{5} / \mathrm{ha}$ & $141.39 \mathrm{a}$ & $140.83 a$ & $111.78 \mathrm{~b}$ & $170.17 \mathrm{a}$ & $145.83 \mathrm{a}$ & $135.61 \mathrm{a}$ \\
\hline $74 \mathrm{~kg} \mathrm{P}_{2} \mathrm{O}_{5} / \mathrm{ha}$ & $145.00 \mathrm{a}$ & $145.56 \mathrm{a}$ & $125.00 \mathrm{a}$ & $178.72 \mathrm{a}$ & $158.44 \mathrm{a}$ & $142.56 \mathrm{a}$ \\
\hline \multicolumn{7}{|c|}{ 4- Total plant fresh wt (g) } \\
\hline $0 \mathrm{~kg} \mathrm{P}_{2} \mathrm{O}_{5} / \mathrm{ha}$ & $175.38 \mathrm{~b}$ & $168.56 b$ & $124.22 \mathrm{c}$ & $198.44 \mathrm{~b}$ & $169.89 \mathrm{c}$ & $167.44 \mathrm{~b}$ \\
\hline $37 \mathrm{~kg} \mathrm{P}_{2} \mathrm{O}_{5} / \mathrm{ha}$ & $189.72 \mathrm{a}$ & $182.50 \mathrm{a}$ & $144.61 \mathrm{~b}$ & $226.00 \mathrm{ab}$ & $193.28 \mathrm{~b}$ & $173.94 \mathrm{ab}$ \\
\hline $74 \mathrm{~kg} \mathrm{P}_{2} \mathrm{O}_{5} / \mathrm{ha}$ & $199.17 \mathrm{a}$ & $193.89 \mathrm{a}$ & $163.61 \mathrm{a}$ & $237.33 \mathrm{a}$ & $210.72 \mathrm{a}$ & $183.11 \mathrm{a}$ \\
\hline \multicolumn{7}{|c|}{ 5- Fresh Leaf / stem ratio } \\
\hline $0 \mathrm{~kg} \mathrm{P}_{2} \mathrm{O}_{5} / \mathrm{ha}$ & $31.65 \mathrm{~b}$ & $28.30 \mathrm{~b}$ & $29.29 \mathrm{a}$ & $32.62 \mathrm{a}$ & $31.76 \mathrm{a}$ & $28.30 \mathrm{a}$ \\
\hline $37 \mathrm{~kg} \mathrm{P}_{2} \mathrm{O}_{5} / \mathrm{ha}$ & $34.69 \mathrm{ab}$ & $29.51 \mathrm{ab}$ & $29.45 \mathrm{a}$ & $33.40 \mathrm{a}$ & $32.74 \mathrm{a}$ & $28.20 \mathrm{a}$ \\
\hline $74 \mathrm{~kg} \mathrm{P}_{2} \mathrm{O}_{5} / \mathrm{ha}$ & $37.73 \mathrm{a}$ & $33.32 \mathrm{a}$ & $30.79 \mathrm{a}$ & $33.41 \mathrm{a}$ & $33.07 \mathrm{a}$ & $28.40 \mathrm{a}$ \\
\hline
\end{tabular}

$1^{\text {st }}$ cut $=60$ days after sowing $\& 2^{\text {nd }}$ cut $=50$ days from $1^{\text {st }} \& 3^{\text {rd }}$ cut $=45$ days from $2^{\text {nd }}$ cut

Table (4): Effect of nitrogenous levels vegetative characteristics of Sudangrass in two growing seasons (2010 and 2011).

\begin{tabular}{|c|c|c|c|c|c|c|}
\hline \multirow{2}{*}{$\begin{array}{l}\text { Season } \\
\text { Cutting No. }\end{array}$} & \multicolumn{3}{|c|}{ Season 2010} & \multicolumn{3}{|c|}{ Season 2011} \\
\hline & $1^{\text {st }}$ cut & $2^{\text {nd }}$ cut & $3^{\text {rd }}$ cut & $1^{\text {st }}$ cut & $2^{\text {nd }}$ cut & $3^{\text {rd }}$ cut \\
\hline \multicolumn{7}{|c|}{ Plant height (cm) } \\
\hline 74 kg N/ha & $195.94 b$ & $178.06 \mathrm{~b}$ & $151.28 \mathrm{~b}$ & $222.50 \mathrm{~b}$ & $211.39 \mathrm{c}$ & $142.22 b$ \\
\hline 121.kg N/ha & $200.28 b$ & $189.50 \mathrm{a}$ & $170.00 \mathrm{a}$ & $239.44 \mathrm{a}$ & $226.11 b$ & $146.11 b$ \\
\hline $171 \mathrm{~kg} \mathrm{~N} / \mathrm{ha}$ & $210.67 \mathrm{a}$ & $192.94 \mathrm{a}$ & $177.50 \mathrm{a}$ & $241.67 \mathrm{a}$ & $235.28 \mathrm{a}$ & $155.28 \mathrm{a}$ \\
\hline \multicolumn{7}{|c|}{ 2- Leaves fresh wt/plant (g) } \\
\hline $74 \mathrm{~kg} \mathrm{~N} / \mathrm{ha}$ & $40.00 \mathrm{~b}$ & $34.17 \mathrm{c}$ & $26.00 \mathrm{c}$ & $41.83 \mathrm{c}$ & $39.50 \mathrm{c}$ & $32.78 \mathrm{c}$ \\
\hline $121 . \mathrm{kg} \mathrm{N} / \mathrm{ha}$ & $46.22 \mathrm{~b}$ & $42.42 \mathrm{~b}$ & $32.56 \mathrm{~b}$ & $56.67 \mathrm{~b}$ & $45.39 \mathrm{~b}$ & $38.06 \mathrm{~b}$ \\
\hline $171 \mathrm{~kg} \mathrm{~N} / \mathrm{ha}$ & $58.06 \mathrm{a}$ & $50.14 \mathrm{a}$ & $39.89 \mathrm{a}$ & $64.28 \mathrm{a}$ & $55.28 \mathrm{a}$ & $44.72 \mathrm{a}$ \\
\hline \multicolumn{7}{|c|}{ 3- Stems fresh wt/plant (g) } \\
\hline $74 \mathrm{~kg} \mathrm{~N} / \mathrm{ha}$ & $127.78 \mathrm{c}$ & $121.94 \mathrm{c}$ & $93.61 \mathrm{c}$ & $131.56 \mathrm{~b}$ & $129.72 b$ & $120.44 \mathrm{~b}$ \\
\hline $121 . \mathrm{kg} \mathrm{N} / \mathrm{ha}$ & $138.89 \mathrm{~b}$ & $135.72 b$ & $110.11 \mathrm{~b}$ & $177.56 \mathrm{a}$ & $146.50 \mathrm{a}$ & $136.83 \mathrm{~b}$ \\
\hline $171 \mathrm{~kg} \mathrm{~N} / \mathrm{ha}$ & $153.33 \mathrm{a}$ & $160.56 \mathrm{a}$ & $130.28 \mathrm{a}$ & $189.89 \mathrm{a}$ & $156.94 \mathrm{a}$ & $151.67 \mathrm{a}$ \\
\hline \multicolumn{7}{|c|}{ 4- Total plant fresh wt (g) } \\
\hline $74 \mathrm{~kg} \mathrm{~N} / \mathrm{ha}$ & $167.78 \mathrm{c}$ & $156.11 \mathrm{c}$ & $119.61 \mathrm{c}$ & $173.39 \mathrm{c}$ & $169.78 \mathrm{c}$ & $153.22 \mathrm{c}$ \\
\hline 121.kg N/ha & $185.11 \mathrm{~b}$ & $178.14 b$ & $142.67 b$ & $234.22 \mathrm{a}$ & $191.89 \mathrm{~b}$ & $174.89 \mathrm{~b}$ \\
\hline $171 \mathrm{~kg} \mathrm{~N} / \mathrm{ha}$ & $211.39 \mathrm{a}$ & $210.69 a$ & $170.17 \mathrm{a}$ & $254.17 \mathrm{a}$ & $212.22 \mathrm{a}$ & $196.39 \mathrm{a}$ \\
\hline \multicolumn{7}{|c|}{ 5- Fresh Leaf / stem ratio } \\
\hline $74 \mathrm{~kg} \mathrm{~N} / \mathrm{ha}$ & $32.16 \mathrm{~b}$ & $28.10 \mathrm{a}$ & $28.22 \mathrm{~b}$ & $32.61 \mathrm{a}$ & $30.53 \mathrm{~b}$ & $27.58 \mathrm{a}$ \\
\hline 121.kg N/ha & $34.01 \mathrm{ab}$ & $31.83 \mathrm{a}$ & $30.56 \mathrm{a}$ & $32.88 \mathrm{a}$ & $31.32 \mathrm{~b}$ & $27.79 \mathrm{a}$ \\
\hline $171 \mathrm{~kg} \mathrm{~N} / \mathrm{ha}$ & $37.91 \mathrm{a}$ & $31.20 \mathrm{a}$ & $30.76 \mathrm{a}$ & $33.94 \mathrm{a}$ & $35.72 \mathrm{a}$ & $29.53 \mathrm{a}$ \\
\hline
\end{tabular}

$1^{\text {st }}$ cut $=60$ days after sowing $\& 2^{\text {nd }}$ cut $=50$ days from first $\& 3^{\text {rd }}$ cut $=45$ days from second cutting. 
Table (5): Effect of seeding rates on vegetative characteristics of Sudangrass in two growing seasons (2010 and 2011).

\begin{tabular}{|c|c|c|c|c|c|c|}
\hline \multirow{2}{*}{$\begin{array}{l}\text { Season } \\
\text { Cutting No. } \\
\end{array}$} & \multicolumn{3}{|c|}{ Season 2010} & \multicolumn{3}{|c|}{ Season 2011} \\
\hline & $1^{\text {st }}$ cut & $2^{\text {nd }}$ cut & $3^{\text {rd }}$ cut & $1^{\text {st }}$ cut & $2^{\text {nd }}$ cut & $3^{\text {rd }}$ cut \\
\hline & \multicolumn{6}{|c|}{ 1- Plant height } \\
\hline $24 \mathrm{~kg}$ seed/ha & $191.93 b$ & $176.07 \mathrm{~b}$ & $149.74 b$ & $217.96 \mathrm{~b}$ & $212.96 b$ & $133.52 b$ \\
\hline \multirow[t]{2}{*}{$36 \mathrm{~kg} \mathrm{seed} / \mathrm{ha}$} & $212.67 \mathrm{a}$ & $197.59 \mathrm{a}$ & $182.78 \mathrm{a}$ & $251.11 \mathrm{a}$ & $235.56 \mathrm{a}$ & $162.22 \mathrm{a}$ \\
\hline & \multicolumn{6}{|c|}{ 2- Leaves fresh wt/plant (g) } \\
\hline $24 \mathrm{~kg} \mathrm{seed} / \mathrm{ha}$ & $56.00 \mathrm{a}$ & $49.94 \mathrm{a}$ & $35.81 \mathrm{a}$ & $60.63 \mathrm{a}$ & $50.15 \mathrm{a}$ & $42.96 \mathrm{a}$ \\
\hline \multirow[t]{2}{*}{$36 \mathrm{~kg} \mathrm{seed} / \mathrm{ha}$} & $40.19 \mathrm{~b}$ & $34.54 \mathrm{~b}$ & $29.81 \mathrm{~b}$ & $47.89 \mathrm{~b}$ & $43.30 \mathrm{~b}$ & $34.07 \mathrm{~b}$ \\
\hline & \multicolumn{6}{|c|}{ 3- Stems fresh wt/plant (g) } \\
\hline $24 \mathrm{~kg} \mathrm{seed} / \mathrm{ha}$ & $158.70 \mathrm{a}$ & $154.07 \mathrm{a}$ & $125.93 \mathrm{a}$ & $189.67 \mathrm{a}$ & $160.74 a$ & $148.22 \mathrm{a}$ \\
\hline \multirow[t]{2}{*}{$36 \mathrm{~kg}$ seed $/ \mathrm{ha}$} & $121.30 \mathrm{~b}$ & $124.74 b$ & $96.74 \mathrm{~b}$ & $143.00 \mathrm{~b}$ & $128.04 b$ & $124.41 \mathrm{~b}$ \\
\hline & \multicolumn{6}{|c|}{ 4- Total plant fresh wt (g) } \\
\hline $24 \mathrm{~kg} \mathrm{seed} / \mathrm{ha}$ & $214.70 \mathrm{a}$ & $204.02 \mathrm{a}$ & $161.74 a$ & $250.30 \mathrm{a}$ & $210.89 \mathrm{a}$ & $191.19 \mathrm{a}$ \\
\hline \multirow[t]{2}{*}{$36 \mathrm{~kg} \mathrm{seed} / \mathrm{ha}$} & $161.48 \mathrm{~b}$ & $159.28 b$ & $126.56 \mathrm{~b}$ & $190.89 \mathrm{~b}$ & $171.33 \mathrm{~b}$ & $158.48 \mathrm{~b}$ \\
\hline & \multicolumn{6}{|c|}{ 5- Fresh Leaf / stem ratio } \\
\hline $24 \mathrm{~kg} \mathrm{seed} / \mathrm{ha}$ & $36.22 \mathrm{a}$ & $32.46 \mathrm{a}$ & $28.30 \mathrm{a}$ & $32.23 \mathrm{a}$ & $31.84 \mathrm{a}$ & $28.88 \mathrm{a}$ \\
\hline $36 \mathrm{~kg} \mathrm{seed} / \mathrm{ha}$ & $33.17 \mathrm{a}$ & $28.29 \mathrm{a}$ & $31.39 \mathrm{a}$ & $34.06 \mathrm{a}$ & $33.75 \mathrm{a}$ & $27.71 \mathrm{a}$ \\
\hline
\end{tabular}

$1^{\text {st }}$ cut $=60$ days after sowing $\& 2^{\text {nd }}$ cut $=50$ days from first $\& 3^{\text {rd }}$ cut $=45$ days from second cutting.

Table (6): Effect of phosphorous rates on forage yield and quality of Sudangrass in two growing seasons (2010 and 2011).

\begin{tabular}{|c|c|c|c|c|c|c|c|c|}
\hline \multirow{2}{*}{$\begin{array}{l}\text { Season } \\
\text { Cutting No. }\end{array}$} & \multicolumn{4}{|c|}{ Season 2010} & \multicolumn{4}{|c|}{ Season 2011} \\
\hline & $1^{\text {st }}$ cut & $2^{\text {nd }}$ cut & $3^{\text {rd }}$ cut & Total & $1^{\text {st }}$ cut & $2^{\text {nd }}$ cut & $3^{\text {rd }}$ cut & Total \\
\hline & \multicolumn{8}{|c|}{ 6- Green forage yield (ton/ha) } \\
\hline $0 \mathrm{~kg} \mathrm{P}_{2} \mathrm{O}_{5} / \mathrm{ha}$ & $27.48 \mathrm{c}$ & $25.63 \mathrm{c}$ & $19.66 b$ & $72.77 \mathrm{c}$ & $27.14 \mathrm{~b}$ & $25.08 \mathrm{~b}$ & $22.22 \mathrm{~b}$ & $74.44 \mathrm{c}$ \\
\hline $37 \mathrm{~kg} \mathbf{P}_{2} \mathbf{O}_{5} / \mathrm{ha}$ & $29.58 \mathrm{ab}$ & $29.53 b$ & $19.02 b$ & $78.13 b$ & $29.58 \mathrm{ab}$ & $26.11 \mathrm{~b}$ & $23.96 \mathrm{~b}$ & $80.19 \mathrm{~b}$ \\
\hline \multirow[t]{2}{*}{$74 \mathrm{~kg} \mathrm{P}_{2} \mathrm{O}_{5} / \mathrm{ha}$} & $34.52 \mathrm{a}$ & $35.12 \mathrm{a}$ & $27.29 \mathrm{a}$ & $96.94 \mathrm{a}$ & $32.25 \mathrm{a}$ & $30.05 \mathrm{a}$ & $26.41 \mathrm{a}$ & $88.71 \mathrm{a}$ \\
\hline & \multicolumn{8}{|c|}{ 1- Crude protein $\%$} \\
\hline $0 \mathrm{~kg} \mathrm{P}_{2} \mathrm{O}_{5} / \mathrm{ha}$ & $6.49 \mathrm{~b}$ & $7.30 \mathrm{~b}$ & $7.34 \mathrm{~b}$ & & $6.74 \mathrm{~b}$ & $7.26 \mathrm{~b}$ & $7.73 \mathrm{~b}$ & \\
\hline $37 \mathrm{~kg} \mathbf{P}_{2} \mathrm{O}_{5} / \mathrm{ha}$ & $7.10 \mathrm{ab}$ & $7.72 \mathrm{ab}$ & $8.32 \mathrm{ab}$ & & $7.33 \mathrm{a}$ & $7.54 \mathrm{ab}$ & $8.41 \mathrm{a}$ & \\
\hline \multirow[t]{2}{*}{$74 \mathrm{~kg} \mathrm{P}_{2} \mathrm{O}_{5} / \mathrm{ha}$} & $7.83 \mathrm{a}$ & $8.13 \mathrm{a}$ & $8.85 \mathrm{a}$ & & $7.55 \mathrm{a}$ & $7.98 \mathrm{a}$ & $8.79 \mathrm{a}$ & \\
\hline & \multicolumn{8}{|c|}{ 2- Crude protein yield (ton/ha) } \\
\hline $0 \mathrm{~kg} \mathrm{P}_{2} \mathrm{O}_{5} / \mathrm{ha}$ & $0.37 \mathrm{~b}$ & $0.36 \mathrm{~b}$ & $0.22 \mathrm{~b}$ & & $0.41 \mathrm{~b}$ & $0.37 \mathrm{~b}$ & $0.29 \mathrm{~b}$ & \\
\hline $37 \mathrm{~kg} \mathrm{P}_{2} \mathrm{O}_{5} / \mathrm{ha}$ & $0.44 \mathrm{~b}$ & $0.45 \mathrm{ab}$ & $0.24 \mathrm{~b}$ & & $0.50 \mathrm{a}$ & $0.41 \mathrm{~b}$ & $0.34 \mathrm{ab}$ & \\
\hline \multirow[t]{2}{*}{$74 \mathrm{~kg} \mathrm{P}_{2} \mathrm{O}_{5} / \mathrm{ha}$} & $0.58 \mathrm{a}$ & $0.59 \mathrm{a}$ & $0.39 \mathrm{a}$ & & $0.56 \mathrm{a}$ & $0.51 \mathrm{a}$ & $0.39 \mathrm{a}$ & \\
\hline & \multicolumn{8}{|c|}{ 3- Crude fiber $\%$} \\
\hline 0 kg $\mathrm{P}_{2} \mathrm{O}_{5} / \mathrm{ha}$ & $53.86 \mathrm{~b}$ & $51.12 \mathrm{a}$ & $44.12 b$ & & $45.97 \mathrm{~b}$ & $42.21 \mathrm{~b}$ & $34.57 \mathrm{~b}$ & \\
\hline $37 \mathrm{~kg} \mathrm{P}_{2} \mathrm{O}_{5} / \mathrm{ha}$ & $62.45 b$ & $53.22 \mathrm{a}$ & $48.39 \mathrm{ab}$ & & $54.06 \mathrm{a}$ & $49.86 \mathrm{a}$ & $42.99 \mathrm{a}$ & \\
\hline $74 \mathrm{~kg} \mathrm{P}_{2} \mathrm{O}_{5} / \mathrm{ha}$ & $69.81 \mathrm{a}$ & $54.37 \mathrm{a}$ & $53.30 \mathrm{a}$ & & $57.38 \mathrm{a}$ & $54.86 \mathrm{a}$ & $47.32 \mathrm{a}$ & \\
\hline
\end{tabular}

$1^{\text {st }}$ cut $=60$ days after sowing $\& 2^{\text {nd }}$ cut $=50$ days from first $\& 3^{\text {rd }}$ cut $=45$ days from second cutting

Table (7): Effect of nitrogenous levels on forage yield and quality of Sudangrass in two growing seasons (2010 and 2011).

\begin{tabular}{|c|c|c|c|c|c|c|c|c|}
\hline \multirow{2}{*}{$\begin{array}{l}\text { Season } \\
\text { Cutting No. }\end{array}$} & \multicolumn{4}{|c|}{ Season 2010} & \multicolumn{4}{|c|}{ Season 2011} \\
\hline & $1^{\text {st }}$ cut & $2^{\text {nd }}$ cut & $3^{\text {rd }}$ cut & Total & $1^{\text {st }}$ cut & $2^{\text {nd }}$ cut & $3^{\text {rd }}$ cut & Total \\
\hline & \multicolumn{8}{|c|}{ 6- Green forage yield (ton/ha) } \\
\hline $74 \mathrm{~kg} \mathrm{~N} / \mathrm{ha}$ & $26.47 \mathrm{c}$ & $25.94 \mathrm{c}$ & $18.21 \mathrm{c}$ & $70.61 \mathrm{c}$ & $24.81 \mathrm{c}$ & $22.96 \mathrm{c}$ & $20.52 \mathrm{c}$ & $68.29 \mathrm{c}$ \\
\hline 121.kg N/ha & $31.28 \mathrm{~b}$ & $29.84 \mathrm{~b}$ & $22.33 \mathrm{~b}$ & $83.45 \mathrm{~b}$ & $29.63 \mathrm{~b}$ & $26.65 \mathrm{~b}$ & $23.85 \mathrm{~b}$ & $80.13 \mathrm{~b}$ \\
\hline \multirow[t]{2}{*}{$171 \mathrm{~kg} \mathrm{~N} / \mathrm{ha}$} & $33.84 \mathrm{a}$ & $34.50 \mathrm{a}$ & $25.42 \mathrm{a}$ & $93.76 \mathrm{a}$ & $35.08 \mathrm{a}$ & $31.63 \mathrm{a}$ & $28.20 \mathrm{a}$ & $94.91 \mathrm{a}$ \\
\hline & \multicolumn{8}{|c|}{ 1- Crude protein \% } \\
\hline $74 \mathrm{~kg} \mathrm{~N} / \mathrm{ha}$ & $6.58 \mathrm{c}$ & $7.17 \mathrm{~b}$ & $7.09 \mathrm{~b}$ & & $6.77 \mathrm{~b}$ & $7.36 \mathrm{a}$ & $7.89 \mathrm{~b}$ & \\
\hline 121.kg N/ha & $7.19 \mathrm{~b}$ & $7.74 \mathrm{ab}$ & $8.58 \mathrm{a}$ & & $7.04 \mathrm{~b}$ & $7.70 \mathrm{a}$ & $8.18 \mathrm{~b}$ & \\
\hline \multirow[t]{2}{*}{$171 \mathrm{~kg} \mathrm{~N} / \mathrm{ha}$} & $7.63 \mathrm{a}$ & $8.24 \mathrm{a}$ & $8.84 \mathrm{a}$ & & $7.81 \mathrm{a}$ & $7.72 \mathrm{a}$ & $8.86 \mathrm{a}$ & \\
\hline & \multicolumn{8}{|c|}{ 2- Crude protein yield (ton/ha) } \\
\hline $74 \mathrm{~kg} \mathrm{~N} / \mathrm{ha}$ & $0.39 \mathrm{~b}$ & $0.40 \mathrm{~b}$ & $0.20 \mathrm{~b}$ & & $0.39 \mathrm{c}$ & $0.38 \mathrm{~b}$ & $0.28 \mathrm{c}$ & \\
\hline 121.kg N/ha & $0.47 \mathrm{a}$ & $0.46 \mathrm{ab}$ & $0.30 \mathrm{a}$ & & $0.47 \mathrm{~b}$ & $0.42 \mathrm{~b}$ & $0.34 \mathrm{~b}$ & \\
\hline \multirow[t]{2}{*}{$171 \mathrm{~kg} \mathrm{~N} / \mathrm{ha}$} & $0.53 \mathrm{a}$ & $0.53 \mathrm{a}$ & $0.34 \mathrm{a}$ & & $0.61 \mathrm{a}$ & $0.49 \mathrm{a}$ & $0.41 \mathrm{a}$ & \\
\hline & \multicolumn{8}{|c|}{ 3- Crude fiber $\%$} \\
\hline $74 \mathrm{~kg} \mathrm{~N} / \mathrm{ha}$ & $65.76 \mathrm{a}$ & $57.75 \mathrm{a}$ & $50.91 \mathrm{a}$ & & $57.82 \mathrm{a}$ & $51.45 \mathrm{a}$ & $45.31 \mathrm{a}$ & \\
\hline 121.kg N/ha & $61.53 \mathrm{a}$ & $51.46 \mathrm{~b}$ & $47.92 \mathrm{a}$ & & $50.24 b$ & $50.25 \mathrm{a}$ & $41.64 \mathrm{ab}$ & \\
\hline $171 \mathrm{~kg} \mathrm{~N} / \mathrm{ha}$ & $58.84 \mathrm{a}$ & $49.51 b$ & $46.97 \mathrm{a}$ & & $49.34 \mathrm{~b}$ & $45.23 \mathrm{a}$ & $37.94 \mathrm{~b}$ & \\
\hline
\end{tabular}

$1^{\text {st }}$ cut $=60$ days after sowing $\& 2^{\text {nd }}$ cut $=50$ days from first $\& 3^{\text {rd }}$ cut $=45$ days from second cutting 
Table (8): Effect of seeding rates $(\mathrm{kg} / \mathrm{ha})$ on forage yield and quality of Sudangrass in two growing seasons (2010 and 2011).

\begin{tabular}{|c|c|c|c|c|c|c|c|c|}
\hline \multirow{2}{*}{$\begin{array}{l}\text { Season } \\
\text { Cutting No. }\end{array}$} & \multicolumn{4}{|c|}{ Season 2010} & \multicolumn{4}{|c|}{ Season 2011} \\
\hline & $1^{\text {st }}$ cut & $2^{\text {nd }}$ cut & $3^{\text {rd }}$ cut & Total & $1^{\text {st }}$ cut & $2^{\text {nd }}$ cut & $3^{\text {rd }}$ cut & Total \\
\hline & \multicolumn{8}{|c|}{ 6- Green forage yield (ton/ha) } \\
\hline $24 \mathrm{~kg} \mathrm{seed} / \mathrm{ha}$ & $28.21 \mathrm{~b}$ & $28.10 \mathrm{~b}$ & $19.42 \mathrm{~b}$ & $75.73 \mathrm{~b}$ & $27.92 \mathrm{~b}$ & $24.85 \mathrm{~b}$ & $21.85 \mathrm{~b}$ & $74.62 b$ \\
\hline \multirow[t]{2}{*}{$36 \mathrm{~kg} \mathrm{seed} / \mathrm{ha}$} & $32.85 \mathrm{a}$ & $32.09 \mathrm{a}$ & $24.55 \mathrm{a}$ & $89.49 \mathrm{a}$ & $31.76 \mathrm{a}$ & $29.31 \mathrm{a}$ & $26.53 \mathrm{a}$ & $87.60 \mathrm{a}$ \\
\hline & \multicolumn{8}{|c|}{ 1- Crude protein \% } \\
\hline $24 \mathrm{~kg} \mathrm{seed} / \mathrm{ha}$ & $7.31 \mathrm{a}$ & $7.79 \mathrm{a}$ & $8.50 \mathrm{a}$ & & $7.27 \mathrm{a}$ & $7.75 \mathrm{a}$ & $8.35 \mathrm{a}$ & \\
\hline \multirow{2}{*}{$36 \mathrm{~kg}$ seed $/ \mathrm{ha}$} & $6.96 \mathrm{~b}$ & $7.64 \mathrm{a}$ & $7.84 \mathrm{a}$ & & $7.14 \mathrm{a}$ & $7.43 \mathrm{~b}$ & $8.27 \mathrm{a}$ & \\
\hline & \multicolumn{8}{|c|}{ 2- Crude protein yield (ton/ha) } \\
\hline $24 \mathrm{~kg} \mathrm{seed} / \mathrm{ha}$ & $0.46 \mathrm{a}$ & $0.45 \mathrm{a}$ & $0.26 \mathrm{a}$ & & $0.48 \mathrm{a}$ & $0.41 \mathrm{~b}$ & $0.31 \mathrm{~b}$ & \\
\hline \multirow[t]{2}{*}{$36 \mathrm{~kg} \mathrm{seed} / \mathrm{ha}$} & $0.47 \mathrm{a}$ & $0.49 \mathrm{a}$ & $0.30 \mathrm{a}$ & & $0.50 \mathrm{a}$ & $0.45 \mathrm{a}$ & $0.37 \mathrm{a}$ & \\
\hline & \multicolumn{8}{|c|}{ 3- Crude fiber $\%$} \\
\hline $24 \mathrm{~kg} \mathrm{seed} / \mathrm{ha}$ & $62.20 \mathrm{a}$ & $53.46 \mathrm{a}$ & $47.05 \mathrm{a}$ & & $56.68 \mathrm{a}$ & $49.66 \mathrm{a}$ & $40.13 a$ & \\
\hline $36 \mathrm{~kg} \mathrm{seed} / \mathrm{ha}$ & $61.88 \mathrm{a}$ & $52.35 \mathrm{a}$ & $50.15 \mathrm{a}$ & & $48.25 b$ & $48.29 \mathrm{a}$ & $43.13 \mathrm{a}$ & \\
\hline
\end{tabular}

$1^{\text {st }}$ cut $=60$ days after sowing $\& 2^{\text {nd }}$ cut $=50$ days from first $\& 3^{\text {rd }}$ cut $=45$ days from second cutting

\section{REFERENCES}

Ahmed, A.G. (2004). Effect of plant density, skipping one irrigation and their interaction growth characters, yield and chemical coposition of grain sorghum. Annals of Agic. Sc., Moshtohor, vol.42 (4): 1473-1485.

Almodares, A.; M. Jafarinia and M.R. Hadi (2009): the effects of nitrogen fertilizer on chemical compositions in corn and sweet sorghum. American-Eurasian J. Agric. \& Environ. Sci. vol.6 (4): 441-446.

Afzal, M.; A.Ahmad, and H.Ahmad (2012): effect of nitrogen on growth and yield of sorghum forage (Sorghum bicolor (1.): Moench cv.). Cercetari Agronomice in Moldova. Vol. xlv, (4)152: 57-64.

Afzal, M.; A.U.Ahmad, S.L. Zamir, F.Khalid, A.U.Mohsin and S.M. Gillani (2013): performance of multicut forage sorghum under various sowing methods and nitrogen application rates. The J. of Animal. \& plant Sci. vol.23 (1): 232-239.

Bahrani M. J., and A.D. Ghenateghestani (2004): summer orge sorghum yield, protein and prussic acid contents as affected by plant density and nitrogen topdressing. J. Agric. Sci. Technol., vol.6: 73-83.

Dorde, G.; S. Jankovic; S. Rakic; R. Maletic; L. Ikanovic and Z. Lakic (2011): effect of nitrogen and harvesting time on chemical composition of biomass of sudangrass, fodder sorghum and their hybrid. Turk. J. Agric. For. Vol.35: 127-138.

Duncan,B.D.(1955): Multiple range and Multiple F. tests. Biometrics, 11: 1-42.

El-Sarag, E. I. and G.M. Abu-Hashem (2009): effect of irrigation intervals and nitrogen rates on forage sorghum under north Sinai conditions. Zagazig J. Agric. Res., vol.36 (1):19-39

Hafiz, S.I. and M.A. El-Kholy (2000): response of two Lupin varieties to folir nutrition with potassium and magnesium under different levels of phosphatic fertilization in sandy soils. J. Agric. Sci. Mansoura Univ. 25 (1): 33-51.

Haggag, M.E.; M.S. Osman; A.M.Rammah and M.E. Mousa (1986): Effect of phosphorus and nitrogen fertilizers on two sorghum varieties. Al-Azhar J. Agric. Res. V1:441-453.

Hassan, K.I.A. (1976): Comparison of nitrogenous and phosphatic needs of forage sorghums in two successive seasons. M. Sc. of Sci. in Agronomy Fac. of Agric. Cairo Univ.

John L. S.; R.L.Randy; E. Raper and B. Schwab (2012): the effect of row spacing and seeding rate on biomass production and plant stand characteristics of non-irrigated photoperiod-sensitive sorghum (Sorghum bicolor (L.) Moench). Industrial crops and Products. Vol 37 (1) May 2012: 527-535

Mahmud, I.A.M.A. (2003): effect of nitrogen and phosphorus on the fodder yield and quality of two sorghum cultivars (Sorghum bicolor L.). International Journal of Agriculture \& biology vol.5 (1):61-63.

Mahmood, A. (2012): performance of sorghum (Sorghum bicolor 1. Moench) as an energy crop for biogas production. Ph.D. in Agric. Fac. of Agric. And Nutritional science Home Economics and Environmental management Justs Liebig University Giessen, Germany.

Sadasivan and Manickam (1991): Biochemical methods for agricultural sciences .p (921 -57)

Yagoub,S.O. and A.K. Abdelsalam (2010): effect of nitrogen and seed rates on growth and yield of forage sorghum (Sorghum bicolor L Moench cv. Abusabien). J.Sci. and Tech. vol.11 (2) March: 4851.

Zahid,S.M.; A.M. Haqqni; M.U. Mufti and S. Shfeeq (2002): Optimiztion of $\mathrm{N}$ and $\mathrm{P}$ fertilizer for higher fodder yield and quality in Mottgrass under irrigation-cum rainfed conditions of Pakistan. Asian Journal of Plant Sciences Vol.1 (6): 690693. 


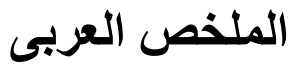 \\ تأثير التسميد الفوسفاتى والازوتى ومعدل التقاوى على محصول العلف الاخضر وجودته فى حشيشة السودان

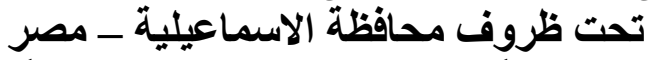

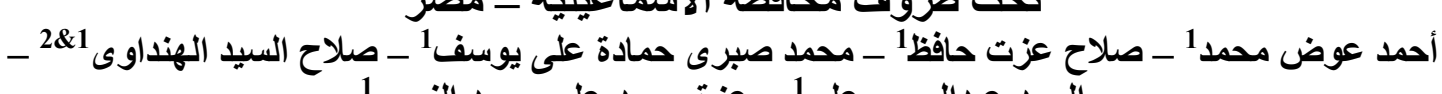

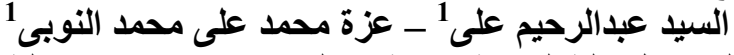

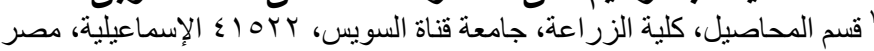

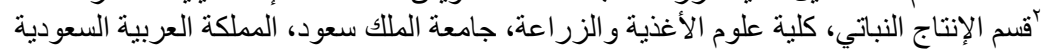

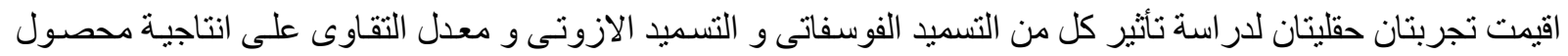

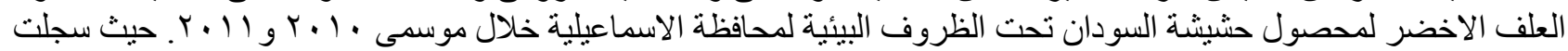

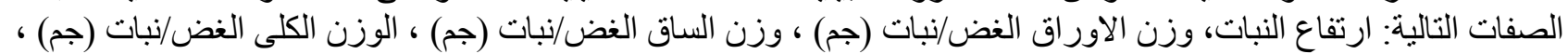

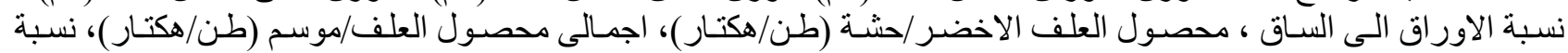

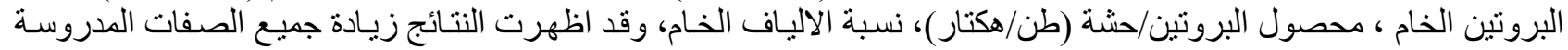

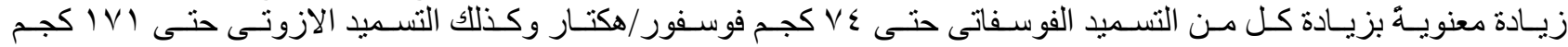

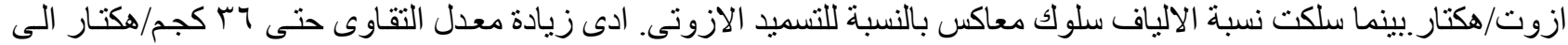

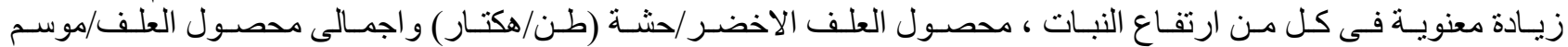

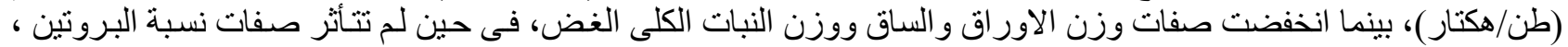

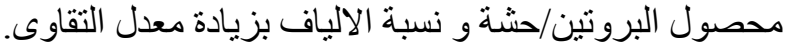
الكلمات المرشـة : حشيشة السودان - السورجم ـ التسميد الفوسفاتى ـ التسميد الازوتى ـ معدلات التقاوى. 